\title{
A stabilized well-balanced RBF-FD meshless method for shallow-water equations.
}

\author{
Joel A. G. Moraes, Eduardo C. Abreu, Luis G. C. Santos.
}

\begin{abstract}
In this work, we are concerned with the study and computing of stabilized radial basis function-generated finite difference (RBF-FD) approximations for shallow-water equations. In order to obtain both stable and highly accurate numerical approximations of convection-dominated shallow-water equations, we use stabilized flat Gaussians (RBFSGA-FD) and polyharmonic splines with supplementary polynomials (RBFPHS-FD) as basis functions, combined with modified method of characteristics. These techniques are combined with careful design for the spatial derivative operators in the momentum flux equation, according to a general criterion for the exact preservation of the "lake at rest" solution in general mesh-based and meshless numerical schemes for the strong form of the shallow-water equations with bottom topography. Both structured and unsructured point clouds are employed for evaluating the influence of cloud refinement, size of local supports and maximal permissible degree of the polynomials in RBFPHS-FD.
\end{abstract}

\section{Key words:}

Well-balanced schemes, Shallow-water equations, Radial basis function-generated finite difference.

\section{Introduction}

Radial basis function-generated finite difference (RBFFD) meshless schemes have been applied in many research areas such as fluid dynamics, heat transfer and seismic modeling, but still, the state of art in this field is far more developed in elliptic problems [1] then in timedependent convection-dominated problems.

However, recent researches [2][3] show that RBF-FD meshless schemes may have an important role on development of stabilized and highly accurate numerical methods for convection-dominated problems.

We apply RBF-FD meshless methods to solve shallowwater equations, satisfying both prevservation of the "lake at rest" solution [4] and stabilization [2] criteria, obtaining highly accurate, stable and conservative solution for this problem.

\section{Results and Discussion}

Both RBFSGA-FD and RBFPHS-FD present good approximations for the solution of shallow-water equations, satisfying "lake at rest" solution.

In both cases, including the variations of supplementary polynomial's highest degree, the approximations are improved as the number of discretization points in point clouds increases, particularly in cases we have unstructured clouds.

Due to the need of constructing new basis, RBFSGA-FD tends to spend significantly longer CPU time than RBFPHS-FD.

\section{Conclusions}

The results show that meshless RBF-FD methods produces very promissing results, satisfying both "lake at rest" and stability conditions and obtainning highly accurate approximattions for shallow-water equations' solution.

Although these methods have been extensively used in another contexts, this work has shown that they may be extent to the context of convection-dominated problems, even attending some consistency conditions, such as the preservation of "lake at rest" solution.

\section{Acknowledgement}

Authors thank PETROBRAS for finnancial support.

[1] SANTOS, L. G. C. et al. Comparing RBF-FD approximations based on stabilized Gaussians and on polyharmonic splines with polynomials. International Journal for Numerical Methods in Engineering.

[2] BENKHALDOUN, Fayssal et al. A stabilized meshless method for timedependent convection-dominated flow problems. Mathematics and Computers in Simulation, v. 137, p. 159-176, 2017.

[3] DEHGHAN, Mehdi; ABBASZADEH, Mostafa. The use of proper orthogonal decomposition (POD) meshless RBF-FD technique to simulate the shallow water equations. Journal of Computational Physics, v. 351, p. 478$510,2017$.

[4] BIHLO, Alexander; MACLACHLAN, Scott. Well-balanced mesh-based and meshless schemes for the shallow-water equations. BIT Numerical Mathematics, p. 1-20, 2017. 